\title{
Novel management of glioma by molecular therapies, a review article
}

\author{
Amin Alinezhad (1), Fatemeh Jafari (2) \\ (1) Department of Clinical Pharmacy, School of Pharmacy, Tehran University of Medical \\ Sciences, Tehran, Iran; (2) Information Technology Engineering, Qazvin Branch, Islamic Azad \\ University, Qazvin, Iran
}

This article is distributed under the terms of the Creative Commons Attribution Noncommercial License (CC BY-NC 4.0) which permits any noncommercial use, distribution, and reproduction in any medium, provided the original author(s) and source are credited.

\begin{abstract}
The most frequent type of brain tumors is Glioma which commonly appears initially in the neuroglia in the central nervous system. They grow steadily and generally do not outspread to neighboring tissue of the brain. By applying dominant remedial regimens, the patients would have negligible survival rates. Despite the achieved advances in conventional glioma therapy, it proved that a proper medication for glioma is not easily reachable. The glioma penetration nature and accumulate resistance considerably limit the remedial options. Superior explanation of the glioma complex pathobiology and characterization of biological proteogenomic may finally open new approaches for the outlining of extra artificial and impressive combination regimens. This aim could be achieved by exclusively outfitting advanced techniques of neuroimaging, terminating synthesis of DNA via genes that activated via prodrugs, experimental technique of gene therapy via conciliating genes of gliomagenesis, targeting miRNA-mRNA activity of oncogenic, applying stem cell therapy for combining inhibitors of Hedgehog-Gli, adaptive transmission of chimeric immunoreceptors $\mathrm{T}$ cells, incorporate inhibitors of regulators of the immune system with conventional remedial modalities and additionally using tumor cell lysates as sources of antigen for efficient evacuation of particular stem cells of tumor via cytotoxic $\mathrm{T}$ lymphocytes. Consequently, in this study the authors trying to survey the latest progressions related to the molecular procedures connected with the formation of glial tumors in addition to the radiation, surgery and chemotherapy limitations. Additionally, the novel strategies of molecular remedies and their procedure for the prosperous treatment of glioma will be discussed.
\end{abstract}

Key Words: Glioma, Gene therapy, Cancer stem cells, Immunotherapy.

Eur J Transl Myol 29 (3): 202-209, 2019

In a diffuse manner permeating gliomas are the central nervous system tumors initial tumors that are categorized into different entities in according with the resemblance of histopathology of the genotype and the tumor cells. Based on the modified fourth edition of the world health organization the classification of tumors consists of 3 main subtypes, spread astrocytoma with the isocitrate dehydrogenase gene mutation (IDH1, IDH2; IDHmt), IDH wild-type of astrocytoma, and IDH mutable or oligodendroglioma and $1 \mathrm{p} / 19 \mathrm{q}$-codeleted. It's while in the inexistence of genetic information into oligodendroglioma or astrocytoma the specification of this information is not possible. ${ }^{1}$ The initial recognition of gliomas often could be done at the period of the late 20 s to the mid-40s it's while in some patients it may be distinguished after the age of 60 years. ${ }^{2}$ Anyway, they are comparatively younger than patients who affected via glioblastoma or anaplastic astrocytoma as a rare malignant tumor of the brain. Most of the patients who are affected commonly will be misdiagnosed with seizures that occasionally might have been unrecognized for a long period of time. Due to the fact that magnetic resonance imaging (MRI) or/and computed tomography have been broadly applied in the detection of various disease, a lot of patients are distinguished with a doubtful glioma for irrelevant symptoms like migraine, head injuries or vertigo. Anyway, the initial pattern of glioma is commonly normal on MRI. Approximately all of them are located above the tentorium cerebelli (supratentorial) and seem to be hyperintense on $\mathrm{T} 1$ with no more contrast and with high intensity on T2/FLAIR. The main location of the lesion is commonly within in the central nervous system white matter. ${ }^{3}$ Approximately $70 \%$ of cancers of brain and $20 \%$ of cancers of spinal cord are glial cell specific. ${ }^{4}$ The factors that would be considered as hazard of production neuroglia tumorigenesis are include viral 


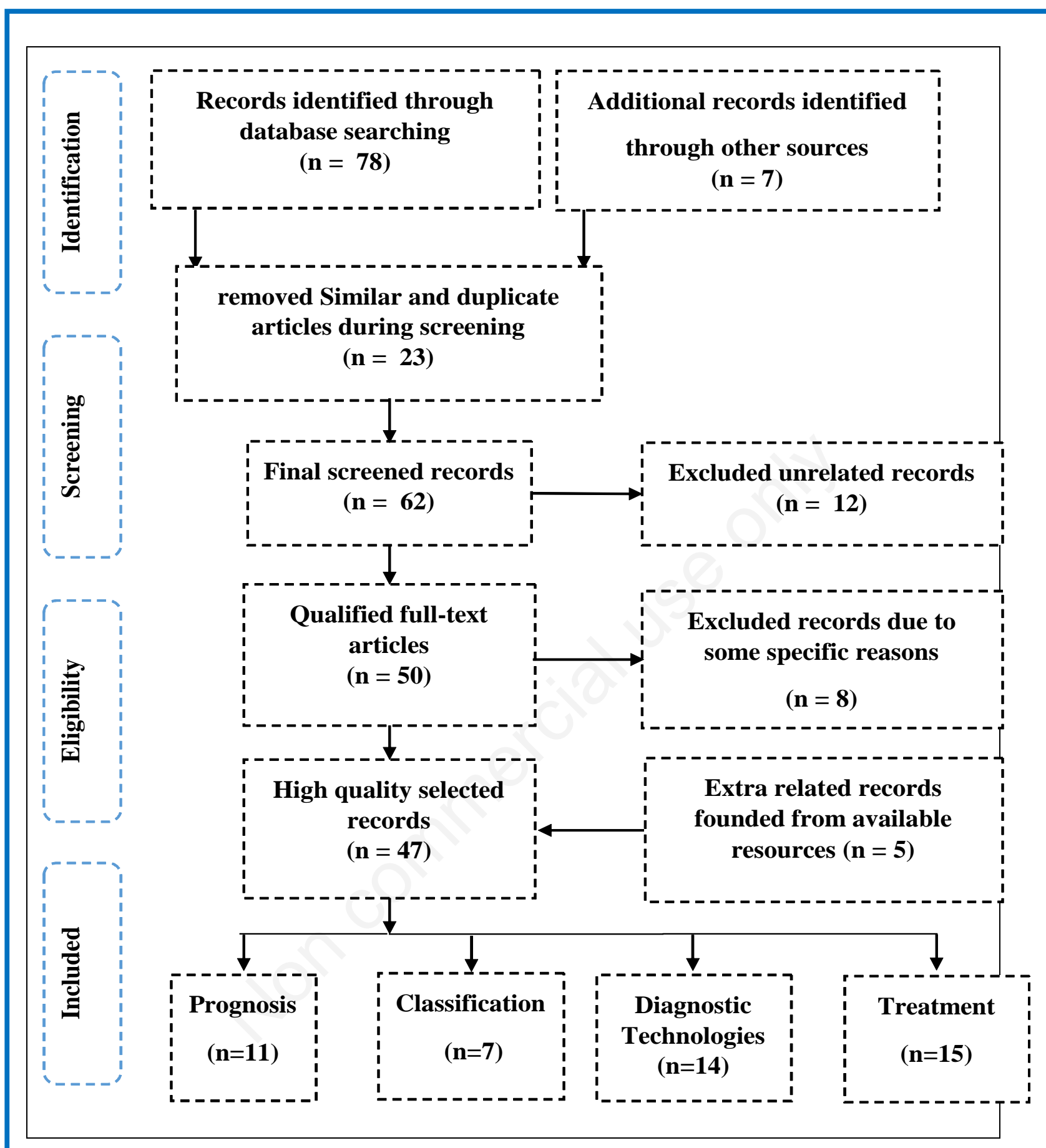

Fig 1. The selection procedure chart of records related to current study in according with PRISMA technique.

infection, ionizing radiation, contact with chemicals, genetic modification (histone, epidermal growth factor receptor (EGFR), cyclin dependent kinase inhibitor $2 \mathrm{~A}$ (CDKN2A), tumor protein 53 (TP53), TACC, IDH, phosphatase and tensin homolog (PTEN), etc.). ${ }^{5}$ The main characterizations of glioma are tumor recurrence, potential in proliferative growth, behavior of infiltrative growth and high degree of intratumor genetic heterogeneity. Knowledgeable information about the size and location of glial tumors are essential scientific factors that could supply a powerful implementation and monitoring of a suitable remedy regimen. The conventional remedial methods for glioma are radiation, chemotherapy and surgery. These remedial techniques are not adequate in comparison to the diverse glioma progression ways. Additionally, segmentectomy is not 
effective too, because of tumors recurrence, acute disease incidence and ventriculoperitoneal shunting requirement. ${ }^{6}$ Radiotherapy is a palliative care that aimed to prevent symptoms instead of curing normal tissue toxicity and resistance. The treatment of cancer by applying radiotherapy may cause loss in potential of neuroregeneration. It's while chemotherapy proved to be a standard remedial method that applied after surgery in company with radiotherapy. Dependent upon the various growth factors roles that are effective in development of brain tumor transforming growth factor (TGF), Ciliary neurotrophic factor (CNTF), fibroblast growth factors (FGF), epidermal growth factor (EGF), vascular endothelial growth factor (VEGF), Platelet-derived growth factor (PDGF), insulin-like growth factor (IGF), etc.) chemotherapy regimen that apply one or more anticancer drugs with, could be designed as an effective remedial method. ${ }^{7}$ Anyway, by crossing the blood brain barrier, fundamental interaction with anticonvulsant remedies or biological steroids and inherent resistance are constrictive factors for applying chemotherapy. The aim of this review is to briefly summing up existing remedial regimens and their dominant limitations for glioma and opening a broad view to the development of new remedial concerns for glioma including unusual strategies like recognition of molecular targets starting off metastasis to restrict glioma. ${ }^{8}$

\section{Methods}

The main aim of this article is to establish a general review of the published literature in the recent years to confer the current situation of the novel molecular therapies for treatment of glioma. So, by application of search terms Glioma, Gene therapy, Cancer stem cells, Biological immunotherapy and Novel molecular therapies we recognized related articles were found. Five comprehensive medical databases such as Medline, PubMed and The EMBASE, The Cochrane Library, Google Scholar and some extra journals were reviewed and relevant article were selected by high precision.

\section{The formation and progress of gliomas}

The infection role in formation of malignant glioma is still suspicious and on the other hand there is a suspicion about the role of infections caused by viruses in occurrence of malignant glioma. Due to the fact that there is not any adequate information about the effective fators on occurrance of malignant glioma, the epidemiologic assessment of viral infections role in glioma etiology is essential. Cytomegalovirus (CMV) is a genus of herpes simplex virus that could be found in malignant tumors. Cobbs et al, ${ }^{9}$ and Lehrer, ${ }^{10}$ lately have been cited that the incidence of cytomegalovirus infection and malignant glioma are reciprocally associated with social and economic status. However, the malignant glioma and CMV association required to be dissolve. The schematic diagram illustrated in Figure 1 clearly exhibits the methodology of current study. Pundole et al, ${ }^{11}$ have assessed the role of varicella zoster virus in glioma risk based on current knowledge and future directions and their study outcomes accentuated the analogy of immunity biomarkers and varicella zoster virus (VZV) infection and with levels of anti-varicella zoster virus IgG for further trials. This neurotropic virus which are capable of infecting nerve cells generally invades the human dorsal root ganglion neurons and causing variations in the VZV proteins seroprevalence such as IE63 and VZV ORF2p proteins. ${ }^{11}$ However, the examination of DNA virus, the first step of Ribonucleic acid (RNA) and protein expression and markers of cellmediated immunity is necessary to dissolve the glial tumorigenesis and infections association.

\section{Clinical factors of glioma prognosis}

The treatment outcomes of glioma patients might be very changeable, lasting from 2 years to over 15 years. Therefore, the recognition of prognostic factors is censorious for appropriate remedial management of the patients. ${ }^{12}$ One the main prognostic scores that is existing for identification patients with risk of being affected via glioma in various grades is derived from randomized studies of European organization for research and treatment of cancer. When multivariate analysis (MVA) is applied, astrocytic as commonest type of cancer of the brain, size of tumor when is higher than six $\mathrm{cm}$, the patient age at least forty years, tumor involves the middle of organ and neurological deficiency at recognition are the main factors of prognosis. ${ }^{13}$ One of the main conventional imaging techniques in prognosis of gliomas is MRI, that is broadly accessible and provides crucial information about any organ anatomy.

\section{CNS tumor histology and grading}

The tumors of central nervous system (CNS) are the superlative destructive kind of cancers because they mostly affect the spinal cord or brain cells that are mainly essential in controling neurological balance. ${ }^{14}$ Because of abnormal inheritance development of these tumors they could easily affect people in all ages. The effect of tumors of brain on patients could be vary via differentiation in age, race and sex. The various cells that are captivated in CNS tumors are Schwann cells, Ependymal cells and glial cells. Glioma a tumor that occur among glial cells which surround cell that transmitting nerve impulses and support and insulate them. About $30 \%$ of CNS tumors and cancers of brain are glioma and $80 \%$ of all glioma tumors are malignant. The grading system of WHO is depend on mitosis, necrosis, atypia, proliferation and endothelial. In according with WHO classification tumors which don't have any of these specifications are within grade I and those have any of these specifications are grading as II. ${ }^{15}$ However, the tumors of grade I and II are contemplated as benign. It's while tumors of grade III are malignant and tumors of grade IV known as glioblastoma multiforme (GBM) are most aggressive 
cancer that starts off in the brain. Additionally, it must be pointed out that, astrocytoma benign tumors are generally occurring among children and tumors of high grade mostly occur among adults. ${ }^{16}$ In the WHO system histological typing of CNS tumors was carried out by describing the existence, variant and characteristics of tissue pattern. The group of tumor which founded integrity of clinicopathological features, where the origins of cellular life were derived was admitted as common and set up the related section subtitles in the booklet of WHO, and this group was called entity. Another group of tumor which was the property of an entity, was a main identity from a molecular, morphological and clinical aspect and in the booklet of WHO was named a variant. And one extra group of tumor with an original morphological characteristic, was similar to other existent group of tumors and in general in the booklet of WHO was named as tissue pattern. The grading of tumors had done in relation to WHO histological and cytological criteria and was performed based on principles of histopathology that essentially could characterize malignancies and also could easily predict the patient clinical course. ${ }^{17}$

\section{Glioma cells classification}

The large-scale study of proteins that known as Proteomics is progressively utilized in both oncological and neurological researches to furnish awareness about disease molecular foundation. More than $50 \%$ of brain tumors are gliomas and malignant gliomas. The application of proteomic technology has recognized modified protein statement but without stability of these modifications being established. As shown by previous studies an analyses of multiple independent proteomic of glioma has showed variations of 99 various proteins. ${ }^{18}$ Classification of glioma is based on large scale study in the omics field and is trying to get integrative view around the biology of glioma. The ordinary approaches that are utilized in proteomics of glioma are protein separation and enrichment, preparation of tissue, quantification, data analysis and mass spectrometry. ${ }^{19,20}$ Glioma of low grade is more frequent among children, whilst among adults, gliomas of higher grade are common. High grade gliomas are infrequent among children but have the same depressing prognosis like adults. In relation with histopathological matters, gene signature, genetic disorder and clinical behavior are alike to WHO grade IV glioblastomas. The large part of pediatric gliomas is benign, slow growing abnormal changes which categorized as grade I or II based on WHO classification of tumors of CNS. This kind of tumors fundamentally rise up within the pons, supratentorial regions and midline constructions including spinal cord, cerebellum and thalamus. ${ }^{21-25}$ The aforementioned systematic factors play a supreme role in the implementation and monitoring of a suitable remedial regimen. Frequently symptoms that are reported for tumors which placed within or under cortical layers are seizures or central neurological or headache variations that causing visuospatial/hemiparesis or hemiplegia alteration. In addition, the pressure inside the skull may increase because of perilesional oedema. ${ }^{26}$

\section{Role of improvements in diagnostic technologies}

Advances in technologies that are concerned with the diagnosis of illness plays a substantial role in comprehension on molecular biology and genetics of glioma as foundation of remedial developments and also assessment of their effectiveness. The diagnostic molecular techniques are applied for detection of brain tumors biomarkers and some of this biomarkers could be used as diagnostics tools for remedial developments which illustrates the existing overlap among them. ${ }^{27}$

\section{Molecular diagnostics}

The techniques of molecular diagnosis are mostly applied for analyzing biomarkers in the proteome and genome. This technique is applied to monitor and diagnose disease, discover risk and to determine which treatment will appear more efficient for each patient. ${ }^{28}$ Molecular investigation of genetic permanent alteration in astrocytomas brain cancers have been performed for recognizing passages which leading to malignant glioma. Molecular diagnostics is a principal foundation for expanding personalized treatment of malignant glioma. ${ }^{29}$ The commonest single gene disorders which caused by DNA changes are due to reinforcement of EGFR, mutation of p53 tumor suppressor transcriptional activator, the CDKN2a gene homozygous elimination and the phosphatase and tensin homolog (PTEN) gene mutation on chromosome 10q23 deletion syndrome. In spite of the fact that most often chromosome abnormalities are when chromosomes 10 and $9 \mathrm{p}$ get losses and chromosomes 7 and 19 gained mutually, the trials for assessment of these genetic alterations have performed as malignant glioma molecular biomarkers too. ${ }^{30}$ Nuclear magnetic resonance allowed to identify oncometabolite 2-hydroxyglutarate which activates the mTOR signalling and produced during tumors mutation in the Isocitrate dehydrogenase enzyme. Choi et al, ${ }^{31}$ by studying around prospective longitudinal analysis of 2hydroxyglutarate magnetic resonance spectroscopy identifies broad clinical utility for the management of patients with IDH-mutant glioma demonstrated that $2 \mathrm{HG}$ concentration is positively associated with tumor cellularity and vary remarkably between low and high grade gliomas. Wang et al., ${ }^{32}$ have worked on detection of tumor-derived DNA in cerebrospinal fluid of patients with primary tumors of the brain and spinal cord and cited that one of the main sources of cancer biomarkers is cell-free DNA (cfDNA) which is perfused by cancer cells. It's while in CNS tumors DNA cannot be easily discovered via testing blood. Anyway, in accordance with their reports by application of patient functional mutations as biological markers, perceptible tumor DNA 
in cerebrospinal fluid levels were recognized in about $70 \%$ of tumors of brain like malignant glioma.

\section{Molecular indicators of prognosis}

The latest progression in gliomas molecular characterization makes possible to have intelligence in evaluating their etiology that is indicated in the novel WHO classification. ${ }^{3}$ IDH1/2 mutation is the spread astrocytoma hallmark and oligodendroglioma primary brain tumours and are correlated with further consequences in contrary to IDH wild-type tumors of astrocytoma that is the most frequent type of glioma recognized in children. ${ }^{33}$ The most frequent recognized mutant is IDH1 R132H that is a principal indication of survival in patients who affected via gliomas and demonstrates over than $90 \%$ of IDH1 and 2 mutations and easily could be recognized via immunehistochemistry. ${ }^{34}$ The recognized mutations are mutations in which the altered gene product possesses a new molecular function and vary the normal activity of enzyme catalysis to to generate alpha-Hydroxyglutaric acid in tumors. This small molecule metabolite that known as oncometabolite could easily prevent Alphaketoglutarate-dependent hydroxylases, such as TET2 that is often mutated in myeloproliferative neoplasms. ${ }^{35}$ So, mutants of IDH appear to intercede the configuration of $\mathrm{CpG}$ islands methylator phenotype as subset of colorectal cancers, ${ }^{36}$ corresponding with wide range variation of gene expression, like genes that suppress cancerous tumors.

\section{Strategies and their restrictions in glioma treatments}

The treatment of glioma requires interdisciplinarity remedial techniques that containing monitoring and diagnosis of states of aggressive malignant. When tumors are in low grade, the recurrence possibility must be monitored, while in tumors with higher grades, derivative recurrence that are resulted from remedy stimulated alterations like radiosurgery should be tracked by monitoring. For individuals with multi-system genetic disorder who are younger than 20 years, modeling monitoring of age dependent diseases must be done every two years. ${ }^{37}$ Glial tumors that are permanent doesn't need monitoring, but glial tumors which are growing should be monitored continuously. Additionally, tumors which are bigger than one $\mathrm{cm}$ must be MRI scanning every six months. Alafaci et al., ${ }^{38}$ have worked on modern neuroimaging techniques in the diagnosis of brain tumors and cited that throughout treatment period of time, brain imaging techniques are applied for diagnosing and assessment of location, scope/size and tumor biological activity. The primary tumors discovery is a delicate job because it's related symptoms are rare. Campen and Porter, ${ }^{39}$ and Ahmed et al., ${ }^{40}$ had worked on current perspectives in diagnosis malignant gliomas and cited that patients commonly report orthostatic headaches, transient visual loss, focal symptoms exacerbation, seizures exacerbation followed by lethargy, vomiting, diplopia and nausea. Traditional remedial regimens are such as surgery, radiotherapy and chemotherapy. The mentioned strategies of treatment are not adequate by comparison with the cancer progression versatile avenues. In most cases cutting off the tumors surgically and alternative neuroimaging could easily help in management of glioma. Applying surgical intervention could easily eliminate hydrocephalus. The partial removal of tumor by surgery is not generally successful because of the recurrence of tumor, the requirement cerebral shunting and acute morbidity. Roa et al., ${ }^{41}$ cited that surgical excision is commonly performed after tumor fractionated radiotherapy. The combination of radiotherapy with surgical excision had proved having better consequence in controlling glioma. But unfortunately radiotherapy has some drawbacks such as obtain radioresistance and normal tissues damages. Anyway, choosing a suitable remedial regimen for proper management of glioma is not much easy, especially when there is a confusion about the adequate selection among chemotherapy and surgery. Gliomas represent high VEGF and compressed vasculature with dense capillary arcades.

\section{Molecular alteration in glial carcinogenesis}

The more dangerous factors that could be considered as the source of glioma includ exposure to toxins like vinyl chloride monomer, electromagnetic radiation (EMR), ionizing radiation, being infected via simian vacuolating virus 40, chromosome mutation and monogenetic disorders. The literature shows that various genes like EGFR, PTEN, CDKN2A and TP53 are fundamentally mutated in glial carcinogenesis. ${ }^{42,43}$ It has been proved that TP53 biological mutation happens in astrocytoma tumors, whilst EGFR reinforcement and PTEN biological mutation are the distinctive characteristics of gliomas in higher grades. ${ }^{44}$ Generally various mechanisms of genetic could cause multiplex mutations, that simplify remedial persistence in the tumor cells through different signaling passages. Gliomas in higher grades have a higher burden of mutation and their belonged frequency shows differential optional compression amongst various brain locations like within the primary auditory cortex, midline region, pontine part of the brainstem and thalamic regions. The specific characteristics of low and high grade Gliomas are multiplex genomic phylogenetic signatures accompanied by considerable Single-nucleotide polymorphism (SNV), copy number aberrations (CNA) and structural alternatives. ${ }^{45}$ In pediatric high grade glioma, the inherited genetic characteristic factors are including germinal mutation in silencer tumor protein p53 and NF1 gene that called neurofibromin; NTRK gene fusions that causing development of a tumor; mutation of platelet derived growth factor receptor alpha (PDGFRA); mutation of EGFR; focal amplification of Cyclin D1, Cyclin D2, Cyclin D3, cyclin-dependent kinase 4 and cell division protein kinase 6; mutation of $\mathrm{H} 3$ histone proteins 
and mutation of diffuse midline glioma H3 K27M. Anyway the aforementioned mutants may be utilized as remedial objectives in pediatric high grade gliomas. ${ }^{46}$

\section{Molecular targeting of metastasis-initiating cells}

Glioma is one of the most invasive and vascularized dangerous cancers. In glioma with higher grades the growth of blood vessels from the existing vasculature is associated with the quality of being malign and inversely associated with the survival of patient. The cells of glioma disrupt and disrupt physical obstacle that include cell junctions, extracellular matrices and basement membranes. The misexpression of a wild-type gene product of various members of the zinc-based enzyme that helps proteolysis is a mark in the invasion process. ${ }^{11}$ The potential of migratory all over the structures of brain, the nature of infiltration and fast tumor advancement of glioma cells make the targets elusive for impressive treatment. Furthermore, insufficient outcome of chemotherapy leads studies to more trials around specific pathways of molecules targeting or around proteins which involved in progression of glioma. Accordingly, the glioma cells revulsive behavior can potentially be expeditiously manipulated via molecular targets identification that cause metastasis, and could be attained via a superior glioma biology comprehension. ${ }^{47}$

\section{Conclusions and future directions}

Location of tumor, symptoms and benefits/risks of various treatments, such as surgery, chemotherapy, and radiation therapy, are the parameters that should be considered in clinical management of the glioma patient. The advers ability of glioma cells to move over length spaces via structures of the brain makes them elusive targets of surgical managements and of ongoing expansion of chemical anti-cancer drugs. Instead of trying to control the glioma decampment, interposition which precisely attacks the invading phenotype must be expanded. Anyway, later developments in brain imaging could help in earlier diagnosis and management of glioma. Progressions in non-destructive imaging techniques and a superior comprehension of biology of glioma could enable neurologists to achieve genetic, cellular, molecular and tumor epigenetic cosmetics, i.e., the achieved information could smooth the way for personalized treatment of glioma. The developing nanotechnology, stem cells transplantation, variations in doses of drugs, reducing the severity of suppressing the immune response mechanisms and additionally a superior comprehension of Messenger RNA and microRNA fundamental interactions are several strategies which might assist stratification of high-grade gliomas. A superior comprehension of glioma multiplex biology and molecular targets recognition which starts off metastasis will simplify the expansion of a new category of anticancer remedies that are more effective and safe. Extensive genomic characterization might recognize molecular triggers and help to glioma subgroups molecular classification. New omics studies could simplify the recognition of biological markers and signatures of glioma protein, additionally to possible remedial regimens design. Among others, hope may be provided developing advanced brain imaging techniques, DNA synthesis dissolution via gene-directed enzymeprodrug therapy, pacifying generation of non-neuronal glioma genes, attacking microRNA activity of oncogenic, Hedgehog Gli/Akt suppressor plus radiotherapy and engaging lysates of tumor as sources of antigen for effective elimination of cancer stem cells via cytotoxic T cells. New generation remedies of glioma treatments will be hopefully developed, specifically focusing on early diagnosis and effective management.

\section{List of acronyms}

CDKN2A - cyclin dependent kinase inhibitor $2 \mathrm{~A}$

cfDNA - cell-free DNA

CMV - Cytomegalovirus

CNS - central nervous system

EGFR - epidermal growth factor receptor

IDH1, IDH2, IDHmt - isocitrate dehydrogenase gene

mutation

GBM - glioblastoma multiforme

MRI - magnetic resonance imaging

PTEN - phosphatase and tensin homolog

TGF - transforming growth factor

TP53 - tumor protein 53

VEGF - vascular endothelial growth factor

\section{Authors contributions}

Both authors played role in the conception, study design, data acquisition and analysis, as well as drafting of the manuscript and approval of the final version.

Acknowledgments None. Funding None

\section{Conflict of Interest}

The authors declare no conflicts of interest.

\section{Ethical Publication Statement}

We confirm that we have read the Journal's position on issues involved in ethical publication and affirm that this report is consistent with those guidelines.

\section{Corresponding Author}

Fatemeh Jafari, Information Technology Engineering, Qazvin Branch, Islamic Azad University, Qazvin, Iran Email: fatemehjafari2010@yahoo.com

E-mail of co-author

Amin Alinezhad: draminalinezhad@yahoo.com

\section{References}

1. Louis DN, Perry A, Reifenberger G, et al. The 2016 World Health Organization Classification of Tumors of the Central Nervous System: a summary. Acta Neuropathol 2016;131:803-820. 
2. Hottinger AF, Weber DM, Levivier M, et al., editors., 4th edition. London: Informa Healthcare; 2010. pp. 283-301.

3. Upadhyay N, Waldman A D. Conventional MRI evaluation of gliomas. Br J Radiol 2011;84:S107S111.

4. Mohammadifard M, Ghaemi K, Hanif $\mathrm{H}$, et al. Marshall and Rotterdam Computed Tomography scores in predicting early deaths after brain trauma. Eur J Transl Myol 2018;28:7542.

5. Schwartzentruber J, Korshunov A, Liu XY, et al. Driver mutations in histone H3.3 and chromatin remodeling genes in paediatric glioblastoma. Nature 2012;482:226-31.

6. Konishi Y, Muragaki Y, Iseki H, et al. Patterns of intracranial glioblastoma recurrence after aggressive surgical resection and adjuvant management: retrospective analysis of 43 cases. Neurol Med Chir (Tokyo) 2012;52:577-86.

7. Olson JJ, Nayak L, Ormond DR, et al. AANS/CNS Joint Guidelines Committee. The role of cytotoxic chemotherapy in the management of progressive glioblastoma: a systematic review and evidencebased clinical practice guideline. J Neurooncol 2014;118:501-55.

8. Swartling FJ. Identifying candidate genes involved in brain tumor formation. Review based on the doctoral thesis "Screening for candidate brain tumor genes - Identifying genes that cooperate with Platelet-derived growth factor in glioma development and progression. Upsala J Med Sci 2008;113:1-38.

9. Cobbs CS, Harkins L, Samanta M, et al. Human cytomegalovirus infection and expression in human malignant glioma. Cancer Res 2002;62:3347-50.

10. Lehrer S. Cytomegalovirus infection in early childhood may be protective against glioblastoma multiforme, while later infection is a risk factor. Med Hypotheses 2012;78:657-8.

11. Pundole X, Amirian ES, Scheurer ME. Role of varicella zoster virus in glioma risk: current knowledge and future directions. OA Epidemiol 2014;2:1-6.

12. Claus EB, Black PM. Survival rates and patterns of care for patients diagnosed with supratentorial lowgrade gliomas: data from the SEER program, 19732001. Cancer 2006;106:1358-63.

13. Pignatti F, Van den Bent M, Curran D, et al. Prognostic factors for survival in adult patients with cerebral low-grade glioma. J Clin Oncol 2002;20:2076-84.

14. Chen L, Zou X, Wang Y, et al. Central nervous system tumors: a single center pathology review of 34,140 cases over 60 years. BMC Clin Pathol 2013;13:1-14.

15. Huszthy PC, Daphu I, Niclou SP, et al. In vivo models of primary brain tumors: pitfalls and perspectives. Neuro Oncol 2012;135:1-15.
16. Zhang J, Wu G, Miller CP, et al. Whole-genome sequencing identifes genetic alterations in pediatric low-grade gliomas. Nat Genet 2013;45:602-12.

17. Wen PY, Huse JT. 2016 World Health Organization Classification of Central Nervous System Tumors. Continuum (Minneap Minn) 2017;23:1531-47.

18. Deighton RF, McGregor R, Kemp J, et al. Glioma Pathophysiology: Insights Emerging from Proteomics. Brain Pathol 2010;20:691-703.

19. Suk K. Proteomic analysis of glioma chemoresistance. Curr Neuropharmacol 2012;10:72-9.

20. Niclou SP, Fack F, Rajcevic U. Glioma proteomics: status and perspectives. J Proteomics 2010;73:1823-38.

21. Ren H, Chen XL, Sun GC, et al. Resection of subependymal giant cell astrocytoma guided by intraoperative magnetic resonance imaging and neuronavigation. Childs Nerv Syst 2013;29: 111321.

22. Richard H, Stogner-Underwood K, Fuller C. Congenital oligodendroglioma: clinicopathologic and molecular assessment with review of the literature. Case Rep Pathol 2015; 1-4.

23. Parish JM, Bonnin JM, Goodman JM, Cohen-Gadol AA. Intrasellar ependymoma: clinical, imaging, pathological, and surgical fndings. J Clin Neurosci 2015;22:638-41.

24. Beaumont TL, Limbrick DD, Smyth MD. Advances in the management of subependymal giant cell astrocytoma. Childs Nerv Syst 2012;28:963-8.

25. Adriaensen ME, Schaefer-Prokop CM, Stijnen T, et al. Prevalence of subependymal giant cell tumors in patients with tuberous sclerosis and a review of the literature. Eur J Neurol 2009;16:691-6.

26. Chintagumpala M, Gajjar A. Brain tumors. Pediatr Clin North Am 2015;62:167-78.

27. Jain KK. Handjournal of Biomarkers. 2nd ed. 2017. New York, NY:Springer.

28. Burtis CA, Ashwood ER, Bruns DE. Tietz Textbook of Clinical Chemistry and Molecular Diagnostics. Elsevier. ISBN 2012978-1-45575942-2.

29. Beiko J, Suki D, Hess KR, et al. IDH1 mutant malignant astrocytomas are more amenable to surgical resection and have a survival benefit associated with maximal surgical resection. Neuro Oncol 2014;16:81-91.

30. Vranova V, Necesalova E, Kuglik $P$, et al. Screening of genomic imbalances in glioblastoma multiforme using high-resolution comparative genomic hybridization. Oncol Rep 2007;17:457-64.

31. Choi C, Raisanen JM, Ganji SK, et al. Prospective longitudinal analysis of 2-hydroxyglutarate magnetic resonance spectroscopy identifies broad clinical utility for the management of patients with IDH-mutant glioma. J Clin Oncol 2016;34:4030-9. 
32. Wang Y, Springer S, Zhang M, et al. Detection of tumor-derived DNA in cerebrospinal fluid of patients with primary tumors of the brain and spinal cord. Proc Natl Acad Sci USA 2015;112:9704-9.

33. Yan H, Parsons DW, Jin G, et al. IDH1 and IDH2 mutations in gliomas. N Engl J Med 2009;360:76573.

34. Capper D, Zentgraf H, Balss J, et al. Monoclonal antibody specific for IDH1 R132H mutation. Acta Neuropathol 2009;118:599-601.

35. Dang L, White DW, Gross S, et al. Cancerassociated IDH1 mutations produce 2hydroxyglutarate. Nature 2010;465:966.

36. Turcan S, Rohle D, Goenka A, et al. IDH1 mutation is sufficient to establish the glioma hypermethylator phenotype. Nature 2012;483:479-483.

37. Jiang T, Jia G, Ma Z, Luo S, et al. The diagnosis and treatment of subependymal giant cell astrocytoma combined with tuberous sclerosis. Childs Nerv Syst 2011;27:55-62.

38. Alafaci C, Granata F, Cutugno M, et al. Modern neuroimaging techniques in the diagnosis of brain tumours. Clinical management and evolving novel therapeutic strategies for patients with brain tumors. Intech Open 2013; 55-76.

39. Campen CJ, Porter BE. Subependymal Giant Cell Astrocytoma (SEGA) treatment update. Curr Treat Options Neurol 2011;13:380-5.

40. Ahmed R, Oborski MJ, Hwang M, et al. Malignant gliomas: current perspectives in diagnosis, treatment, and early response assessment using advanced quantitative imaging methods. Cancer Manag Res 2014;6:149-70.

41. Roa W, Brasher PM, Bauman G, et al. Abbreviated course of radiation therapy in older patients with glioblastoma multiforme: a prospective randomized clinical trial. J Clin Oncol 2004;22:1583-8.

42. Sottoriva A, Spiteri I, Piccirillo SG, et al. Intratumor heterogeneity in human glioblastoma reflects cancer evolutionary dynamics. Proc Natl Acad Sci USA 2013;10:4009-14.

43. Parker NR, Khong P, Parkinson JF, et al. Molecular heterogeneity in glioblastoma: potential clinical implications. Front Oncol 2015;5:55.

44. Ducray F, Marie Y, Sanson M. IDH1 and IDH2 mutations in gliomas. N Engl J Med 2009;360:1-9.

45. Berhouma M. Management of subependymal giant cell tumors in tuberous sclerosis complex: the neurosurgeon's perspective. World J Pediatr 2010;6:103-10.

46. Jones C, Baker SJ. Unique genetic and epigenetic mechanisms driving paediatric diffuse high-grade glioma. Nat Rev Cancer 2014;14:651-61.

47. Ouyang $\mathrm{T}$, Zhang $\mathrm{N}$, Benjamin $\mathrm{T}$, et al. Subependymal giant cell astrocytoma: current concepts, management, and future directions. Childs Nerv Syst 2014;30:561-70.

Submission: April, 7, 2019

Revision received: May 17, 2019

Acceptance: May 18, 2019 